\title{
Fail safe design with crack stoppers and warners
}

\author{
C. Mattheck, Ch. Wissner, K. Bethge \& O. Kraft \\ Institute for Material Research II, \\ Forschungszentrum Karlsruhe, Germany
}

\begin{abstract}
Trees attach thicker tree rings at overloaded places. This principle is mimicked here in order to delay crack initiation and propagation. Bulges are attached beside notches to reduce stress concentrations. The bulges have to be shape optimized so as to not cause notch stresses themselves. Brittle coating can act as a warner near notches. The crack stoppers are described by FEM and the result is verified by comparative fatigue testing of prototypes leading to up to 21 times longer fatigue life.
\end{abstract}

Keywords: crack stoppers, crack warners.

\section{Introduction}

The resistance of a mechanical stressed structure against crack initiation is at the one hand a material attribute but on the other hand a matter of the mechanical effective tensions and so directly depending on the shape of the structure.

Former studies showed the great effect of a shape optimization to reduce the maximum stresses and to get a more homogeneous tension distribution. The Computer Aided Optimization (CAO) was inspired by the growth of trees, who attach more material, thicker tree rings, at highly stressed regions. As a complex an iterative computer algorithm, this method achieves very good results, need otherwise special computer programmes and advanced users.

A few years ago with the Method of Tensile Triangles a method was created by Mattheck [2], which everybody can use. Without computers, only with pencil and setsquare. The simple way of construction is shown at figure 1 .

The basic idea of the Method of Tensile Triangles is to find a shape that fits to the flow of forces. This means, that no abrupt changes in the flow of forces 


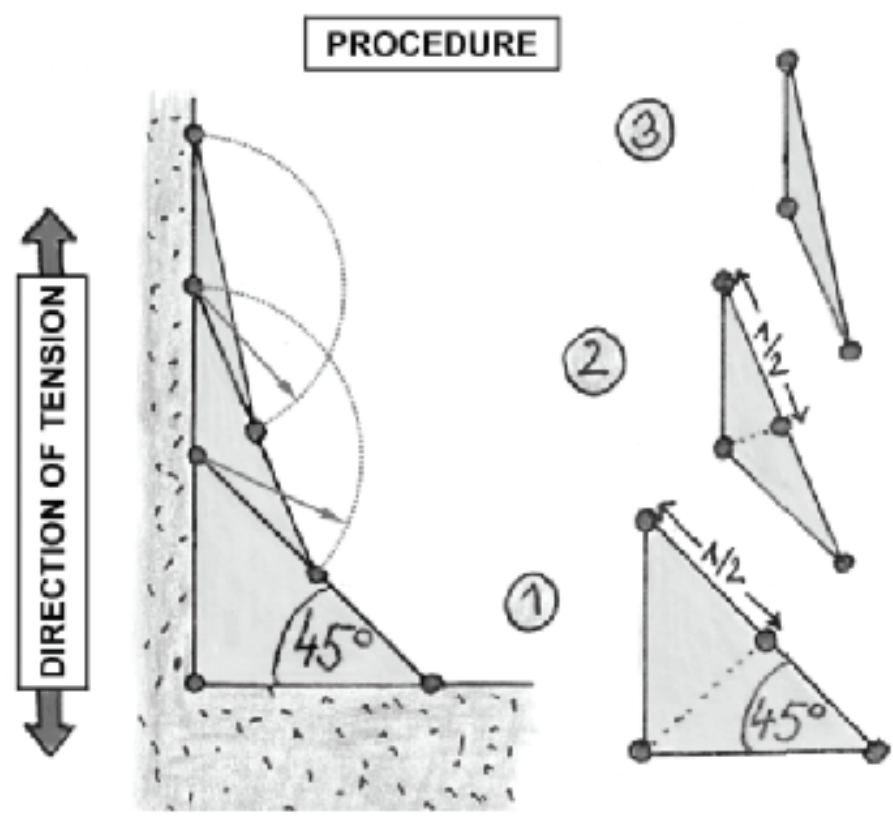

Figure 1: $\quad$ Procedure of the Method of Tensile Triangles [2].

appear. The sharp bend at the bottom of the contour has no mechanical effect, because the maximum angle of the flow of forces is $45^{\circ}$.

Fig. 2 shows a comparison of the Mises stress distribution as a FEM-plot of a "normal" notch (left side) with an optimized one (right side). Both contours have same radial space. The plot on the left side shows a stress peak in the notch.

This notch stress is reduced bye use of tensile triangles contour line.

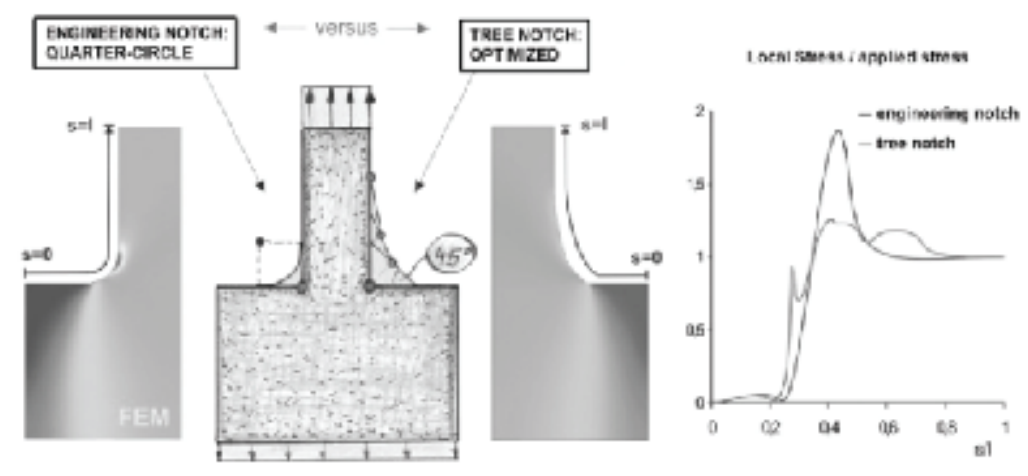

Figure 2: Comparison between a normal, quarter-circle and the optimized notch [2]. 


\section{Crack stoppers}

Other technical structures that are often afflicted by cracks are holes. The cracks are caused by high tensions that effect, when the flow of forces is disturbed by the hole. The cavity of the hole causes also a superposed bending, that produces tensile stress at the interior of the hole.

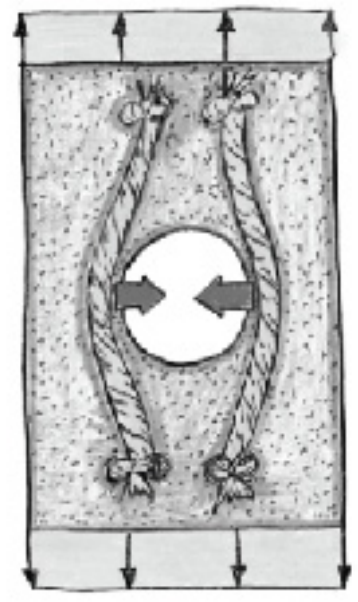

Figure 3: $\quad$ Superimposed bending [2].

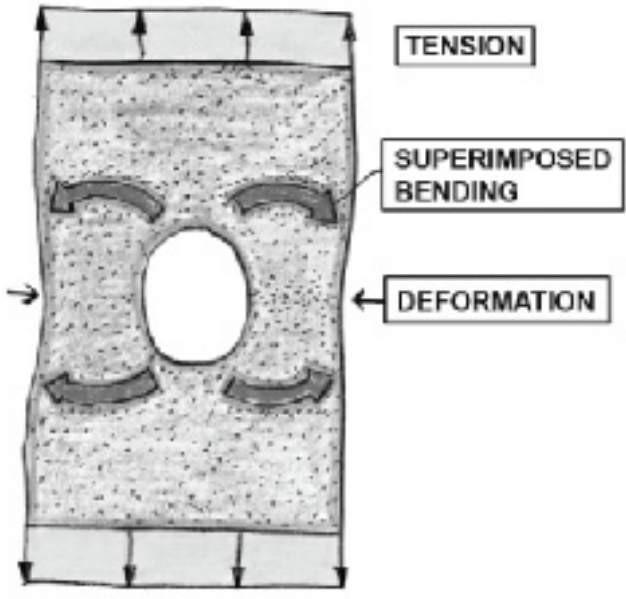

To reduce the tensile stress at the hole two things can be done. At the one hand you can reduce tension by increasing the cross section. A bigger area reduces the tension, while the force keeps equal. This means to use additional material. Otherwise you can constrain the superposed bending by buttressing the hole.

For uniaxial stress condition adding two bulges near the hole minimize the stress concentrations effectively. If these bulges are not shape optimized, new predetermined breaking points occur.

Figure 4 shows prototypes with various shapes of the bulges. At the left side a normal hole without any additional material, in the middle a hole with nonoptimized bulges and at the right side one with shape optimized bulges, using the Method of Tensile Triangles.

These prototypes had failed at the fatigue test after approximately 300.000 and 350.000 cycles respectively. It is clearly to see, that in the middle specimen the crack was initiated at the intersection of the base material and the bulges.

The one with shape-optimized bulges (right) didn't fail at all. This test was stopped after 6.500 .000 cycles.

Figure 5 shows exemplary the longitudinal section of the shape-optimized bulges. With the Method of Tensile Triangles the intersection of the basematerial to the bulges was shape-optimized. 


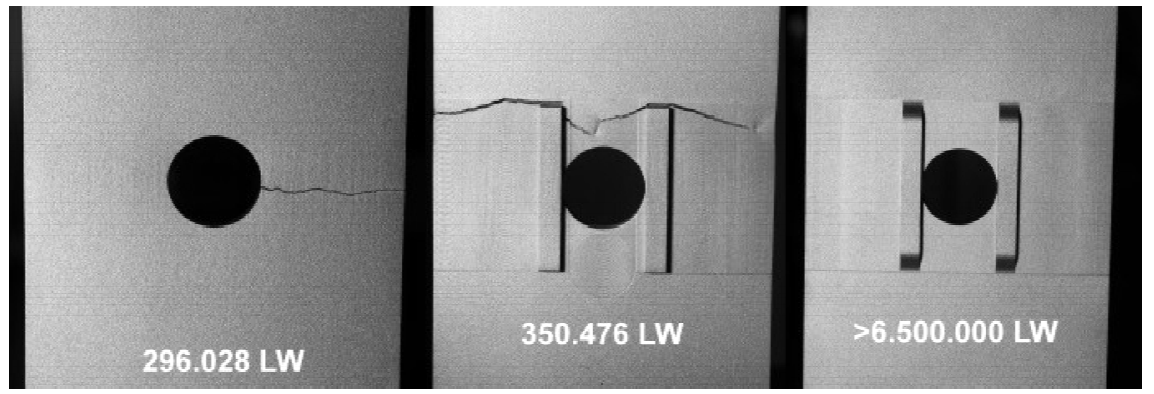

Figure 4: Fatigue-tested specimen with various forms of bulges as crackstoppers.
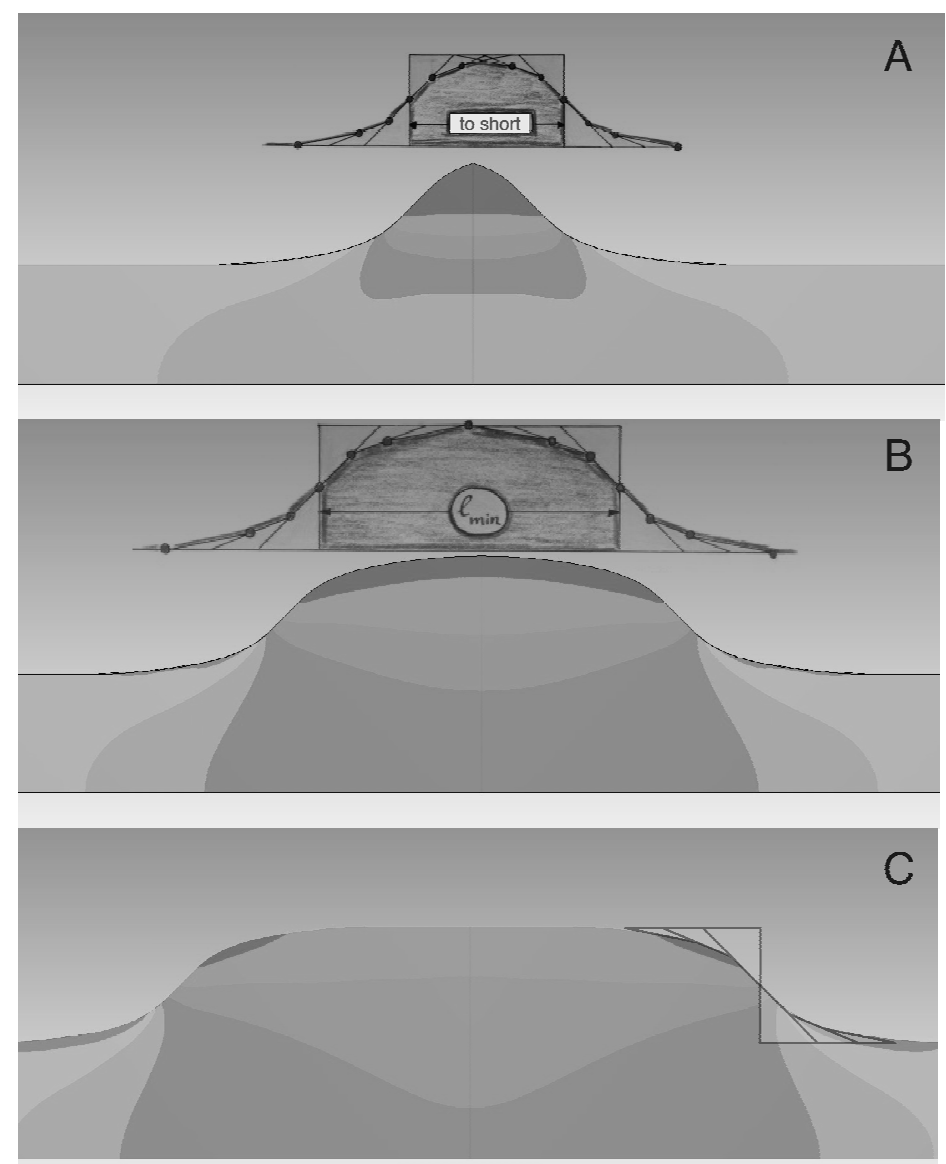

\section{- V. Mises stress +}

Figure 5: Three different longitudinal section of crack-stopper bulges, optimized by the Method of Tensile Triangles. 
An important issue is to know how long the bulges have to be, so that the flow of forces can use the entire, additional material.

Form A shows a huge under loaded area on the top of the bulge. Form B and $\mathrm{C}$ show a more homogeneous tension distribution and the flow of forces make more use of the additional material.

The minimum length is at least two complete arrays of tensile triangles in the convex middle and also another two arrays each at the concave beginning and the end of the bugle.

The upper mentioned examples only show the bulges at uniaxial tension stress. For a biaxial tension stress, there must be an adaptation for the bulges. A universal solution is a bulge-ring, without any preferential orientation. Figure 6 shows an example.

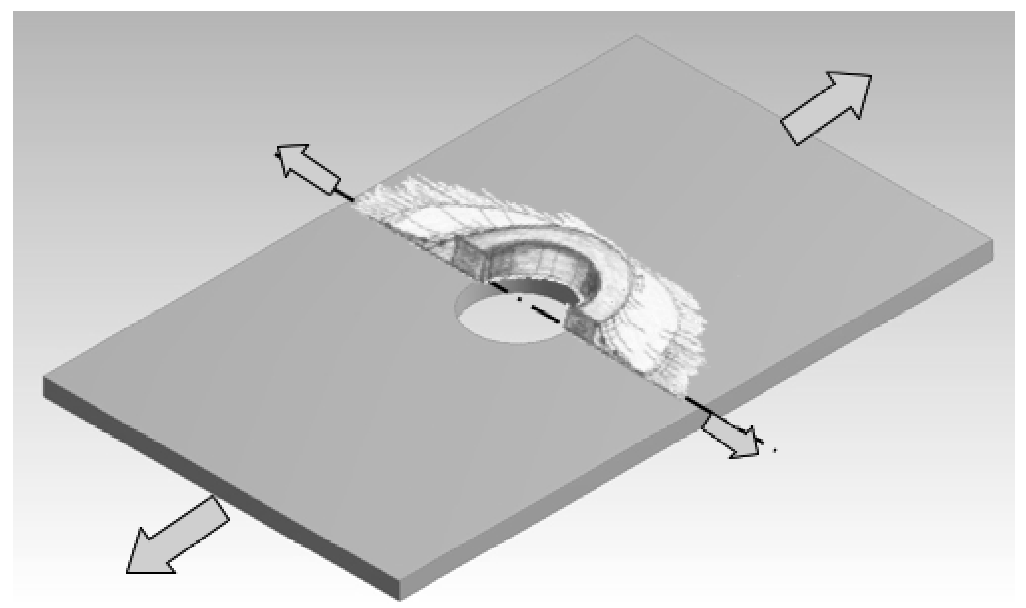

Figure 6: $\quad$ Ring-bulge for multiaxial tension stress.

With these kinds of bulges it is possible to prohibit the initiation of cracks effectively.

\section{Crack warners}

With a small adaptation it is also possible to use this crack-stoppers also as warners.

When the bulge is coated with an extremely brittle varnish, it is possible to detect crack initiation or propagation.

\section{Avoiding all effects of superimposed bending}

Adding material as bulges can reduce the risk of crack initiation, by reducing the tension. As seen in figure 3 the superposed bending can be constrained by buttressing the hole. 


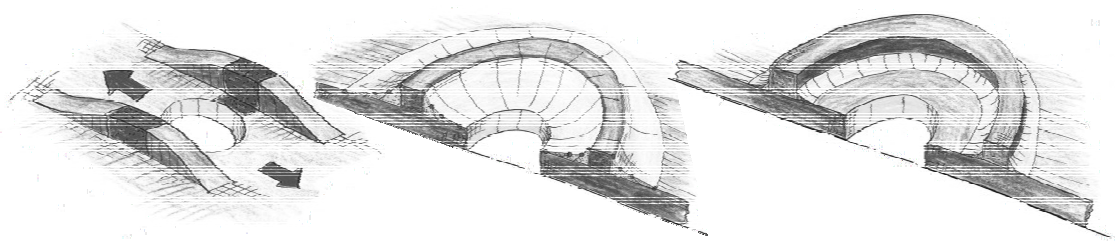

Figure 7: Uni- and multiaxial crack-warners with a small gap between the hole and the bulge.

To avoid all influences of superimposed bending it is necessary to use the additional material and the stiffening of the hole at both sides. If only one side is reinforced new effects of superposed bending might appear.

Figure 8 shows an extremely deformed quarter FEM-model with single-sided crack-stopper. The original shape is shown as wire frame, the whole model top right. The extreme deformation clarifies the superimposed bending.

The additional material on the upper side stiffen it in such way, that superposed bending occurs and the lower side is stressed by additional tensile stress.

This can even causes higher stress than on an equal hole without any bulges.

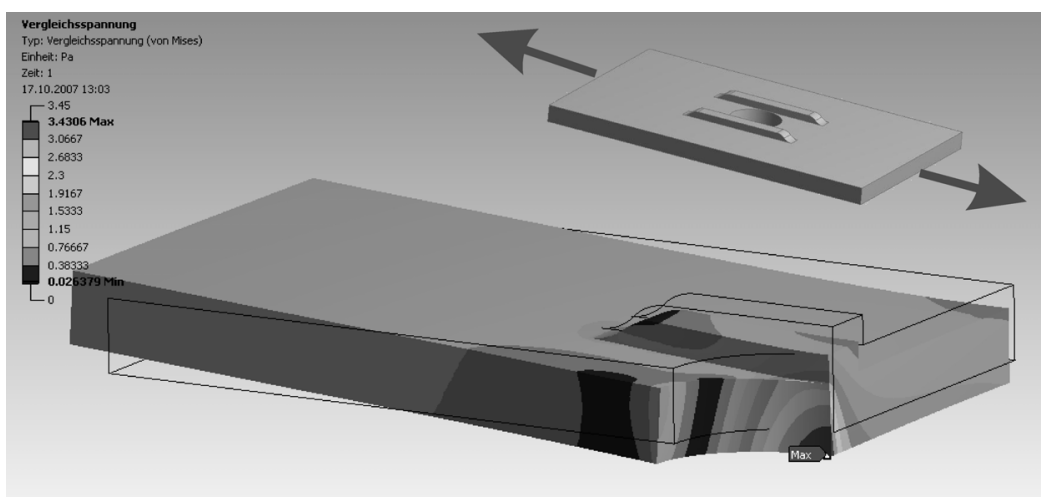

Figure 8: Quarter FEM-model with single-sided bulges under tension load.

\section{Summary}

With bulges or ring-bulges, optimized with the Method of Tensile Triangles, it's possible to improve the resistance against crack initiation and propagation.

These bulges don't need expensive computer work done by advanced users.

The easy and plausible way of the tensile triangles procedure makes this method as simple as powerful. There are many possible fields of application, especially casting, where these methods can improve the resistance against cracks.

Further investigation is needed to explore all effects and influences of the superimposed bending and to improve the practical use of this method. 


\section{References}

[1] Mattheck, C. The face of failure - Form and Failure in Nature and Engineering. www.mattheck.de, Verlag Forschungszentrum Karlsruhe, Karlsruhe, 2004.

[2] Mattheck, C. Secret design rules of nature - optimum shapes without computers. www.mattheck.de, Verlag Forschungszentrum Karlsruhe, Karlsruhe, 2007 (German version: 2006). 